\title{
A Model for Evaluating the Performance of Code Phase Assignment CDMA LAN
}

\author{
F. L. Lo \\ T. S. Ng \\ fllo@hkueee.hku.hk \\ tsng@hkueee.hku.hk \\ T. I. Yuk \\ tiyuk@hkueee.hku.hk \\ Department of Electrical and Electronic Engineering \\ The University of Hong Kong \\ Pokfulam Road \\ Hong Kong
}

Tel.: (852) 2859-2686

Fax.: (852) 2559-8738

\begin{abstract}
This paper describes a model for a fullyconnected, full duplex, and synchronized CDMA LAN where the receivers use the same maximal length sequence with different chip phase offset for receiving messages. ALOHA medium access is used. The performance of the model is analyzed by considering the succession of states the system is in as a Markov process. The behaviour of a 5-station system is shown.
\end{abstract}

\section{INTRODUCTION}

CDMA ALOHA systems using spread-spectrum signals to achieve correct reception of several signals in the same band of frequencies as well as the ALOHA multiple access technique have been studied extensively for use in satellite and cellular communication, e.g., [1], [2]. In this paper, we shall concentrate on the use of CDMA ALOHA for a Local Area Network. Since all stations in a LAN are close together, and even mobile stations do not move fast, it is possible to synchronize all stations to a master clock. The same maximal length sequence can then be used as the spreading code for all stations, with each user allocated a different chip phase, as suggested in [3-5]. If the synchronization is perfect and there is no multipath interference, as in [4], then a phase difference of one chip can be used. Even when there is timing imperfection and multipath dispersion, it has been shown in [3] that Code Phase Assignment (CPA) CDMA systems are still feasible, and the performance of such systems are much better than those using different spreading sequences, as long as the chip phase difference is large enough so that signals from two different stations will not share the same chip phase at the receiver. It must be pointed out that the spread ALOHA described in [5] is different from the approach used here.
In Section II, a simple model is proposed for a slotted CPA LAN and in Section III, this model is analyzed using a discrete time Markov chain. The delay and throughput results are presented in Section IV and the paper ends with some conclusions in Section V.

\section{CPA LAN MODEL}

All the stations are synchronized and each station is assigned a different chip phase of the same maximal length spreading sequence with which it will receive messages. Thus if a station wishes to send a message to station $k$, it will transmit that message with the system spreading sequence having the chip phase of station $k$. Time is divided into slots and each slot is long enough to hold exactly one message or packet. Stations can transmit and receive at the same time, i.e., the links are full-duplex, but if two or more packets are directed to the same destination during the same slot time, the packets will collide and be destroyed.

Packets are generated at each of the stations at the similar rate of $s$ packets/slot independent of other stations and other packets, and each station is equally likely to transmit to all other stations, i.e., uniform traffic matrix is assumed. If a station has no packets to transmit, it is said to belong to the idle mode $T_{0}$, and has an empty buffer to hold a packet. Whenever a packet arrives at an idle station during a slot, the station will attempt with probability one to transmit the packet during the next slot. If no other packets are transmitted to the same receiving station, the packet will get through. Should two or more packets be transmitted to the same destination, all the packets will be destroyed. All stations holding a packet that has been unsuccessfully transmitted are said to be blocked. All blocked stations with packets meant 
for station $k$ are said to belong to the retransmission, or blocked mode $R_{k}$. Blocked stations will not accept new packets that are generated, and will attempt to retransmit with probability $p$ in each slot subsequent to a collision.

All timing imperfections, multipath fading, noise and other sources of interference are neglected in this model, and packets sent that do not collide are assumed to be received accurately. Transmitters are also assumed to know immediately after a slot whether the packet it has sent is involved in a collision.

\section{ANALYSIS OF MODEL}

The number of idle stations in $T_{0}$ is denoted by $n_{0}$ and the number of blocked stations belonging to $R_{k}$ is denoted by $n_{k}$. The system with $N$ users can then be characterized by the state vector $\left(n_{1} n_{2} \cdots n_{N}\right)$. Note that $n_{0}=N-\sum_{k=1}^{N} n_{k}$.

All rearrangements of $n_{k}$ in $\left(n_{1} n_{2} \cdots n_{N}\right)$ will be called substates of the same state and are equally likely since all the stations are statistically identical. For example, $\left(\begin{array}{lll}0 & 0 & 1\end{array}\right)$, $\left(\begin{array}{lll}0 & 1 & 0\end{array}\right)$ and $\left(\begin{array}{lll}1 & 0 & 0\end{array}\right)$ are all of the same state in a 3-user system. Even though any of the substates can be used to represent that state, we shall use the substate where $n_{i}>n_{j}$ if $i<j$ to represent the state. Thus $\left(\begin{array}{lll}1 & 0 & 0\end{array}\right)$ will be used to represent the above 3 substates. The states for systems with any number of users can then be written down easily by listing them systematically, as is demonstrated below for a system with 4 stations:

\begin{tabular}{|c|c|c|}
\hline$\left(\begin{array}{llll}0 & 0 & 0 & 0\end{array}\right)$ & or & (0) \\
\hline$\left(\begin{array}{llll}1 & 0 & 0 & 0\end{array}\right)$ & or & (1) \\
\hline$\left(\begin{array}{llll}2 & 0 & 0 & 0\end{array}\right)$ & or & (2) \\
\hline$\left(\begin{array}{llll}1 & 1 & 0 & 0\end{array}\right)$ & or & $\left(\begin{array}{ll}1 & 1\end{array}\right)$ \\
\hline$\left(\begin{array}{llll}3 & 0 & 0 & 0\end{array}\right)$ & or & (3) \\
\hline$\left(\begin{array}{llll}2 & 1 & 0 & 0\end{array}\right)$ & or & $\left(\begin{array}{ll}2 & 1\end{array}\right)$ \\
\hline$\left(\begin{array}{llll}1 & 1 & 1 & 0\end{array}\right)$ & or & $\left(\begin{array}{lll}1 & 1 & 1\end{array}\right)$ \\
\hline$\left(\begin{array}{llll}3 & 1 & 0 & 0\end{array}\right)$ & or & $\left(\begin{array}{ll}3 & 1\end{array}\right)$ \\
\hline$\left(\begin{array}{llll}2 & 2 & 0 & 0\end{array}\right)$ & or & $\left(\begin{array}{ll}2 & 2\end{array}\right)$ \\
\hline$\left(\begin{array}{llll}2 & 1 & 1 & 0\end{array}\right)$ & or & $\left(\begin{array}{lll}2 & 1 & 1\end{array}\right)$ \\
\hline
\end{tabular}

As an example, the state (2 1) means that 2 stations are blocked trying to communicate to a particular station, and one is blocked trying to transmit to another. The remaining station is, of course, idle. It is easy to see that the number of possible states for an $N$-user system is $n=\sum_{k=1}^{N} p(k)+1-2$, where $p(k)$ is the partition function for the integer $k$, i.e., the total number of ways of adding non-negative integers to form $k$. Thus, since $4=(4)=(3+1)=(2+2)=(2+1+1)=(1+1+1+1)$, it follows that $p(4)=5$. It is necessary to add 1 as the state $(0)$ is possible, and subtract 2 , as the states $(N)$ and $\left(\begin{array}{llll}1 & 1 & \cdots & 1\end{array}\right)$ are not possible since a station will not transmit to itself. Note that each substate may have several configurations. For example, the substate $\left(\begin{array}{lll}0 & 1 & 0\end{array}\right)$ for a 3 -station system may have either user 1 or 3 blocked trying to send a packet to user 2 .

Since the state a system is in at any particular slot time depends only on the state the system was in at the previous slot, the evolution of the system states forms a Markov process. Furthermore, since the state space is finite, equilibrium or stationary probabilities exist for all the states. If we write $\pi_{i j}$ as the stationary probability that the system is in state $i$ substate $j$, or in shorthand form $\{i, j\}$, and $p_{i j, k l}$ as the probability that $\{i, j\}$ will change to $\{k, l\}$ in a slot time, then we can write the system equations in matrix form as

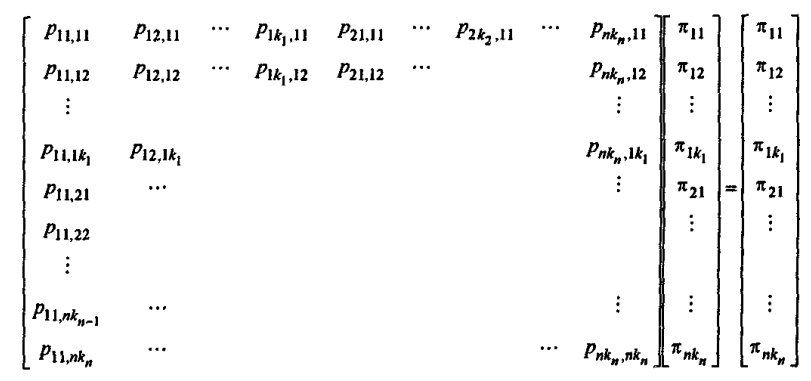

where $k_{1}, k_{2}, \cdots, k_{n}$ are the numbers of substates of state $1,2, \cdots, n$, respectively. Note that $\pi_{11}=\pi_{12}=\cdots=\pi_{1 k_{1}}$ and $\pi_{21}=$ $\pi_{22}=\cdots=\pi_{2 k_{2}}$,etc., since all substates of a certain state are equally probable.

Let us denote the difference of blocked stations in the various modes between $\{i, j\}$ and $\{k, l\}$ by $\left(d_{1} d_{2} \cdots d_{N}\right)$. A little thought will show that these differences in the number of blocked stations between a certain substate, say 1 , of $i$ and all substates of $k$, and another substate of $i$, say 2, and all substates of $k$, are just rearrangements of the same set of $\left(d_{1} d_{2} \cdots d_{N}\right)$. For example, the differences in the number of blocked stations between $\left(\begin{array}{lll}1 & 0 & 0\end{array}\right)$ and all substates of $\left(\begin{array}{lll}2 & 0 & 0\end{array}\right)$

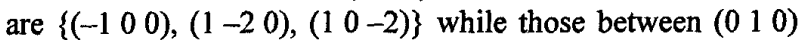
and all substates of $(200)$ are $\left\{\left(\begin{array}{lll}-2 & 10\end{array}\right),\left(\begin{array}{lll}0 & -1 & 0\end{array}\right),\left(\begin{array}{lll}0 & 1 & -2\end{array}\right)\right\}$. Since the probability that a certain $\{i, j\}$ will change to a certain $\{k, l\}$ after a slot depends only on the difference in the number of blocked stations in the various blocked modes, as will be seen later, hence $\left(p_{11,11}+p_{11,12}+\cdots+p_{11,1 k_{1}}\right)=\left(p_{12,11}+\right.$ $\left.p_{12,12}+\cdots+p_{12,1 k_{1}}\right)=\cdots=\left(p_{1 k_{1}, 11}+p_{1 k_{1}, 12}+\cdots+p_{1 k_{1}, 1 k_{1}}\right) \equiv p_{11}$ 
and $\left(p_{21,11}+p_{21,12}+\cdots+p_{21,1 k_{1}}\right)=\left(p_{22,11}+p_{22,12}+\cdots+p_{22,1 k_{1}}\right)$ $=\cdots=\left(p_{2 k_{2}, 11}+p_{2 k_{2}, 12}+\cdots+p_{2 k_{2}, 1 k_{1}}\right) \equiv p_{21}$, etc. Multiplying out the first $k_{1}$ rows of (1) and adding, we get

$\left(p_{11,11}+p_{11,12}+\cdots+p_{11,1 k_{1}}\right) \pi_{11}+\left(p_{12,11}+p_{12,12}+\cdots+p_{12,1 k_{1}}\right) \pi_{12}+\cdots+$

$\left(p_{21,11}+p_{21,12}+\cdots+p_{21,1 k_{1}}\right) \pi_{21}+\left(p_{22,11}+p_{22,12}+\cdots+p_{22,1 k_{1}}\right) \pi_{22}+\cdots+$

$\cdots+$

$\left(p_{n 1,11}+p_{n 1,12}+\cdots+p_{n 1,1 k_{1}}\right) \pi_{n 1}+\left(p_{n 2,11}+p_{n 2,12}+\cdots+p_{n 2,1 k_{1}}\right) \pi_{n 2}+\cdots+$

$\left(p_{n k_{n}, 11}+p_{n k_{n}, 12}+\cdots+p_{n k_{n}, 1 k_{1}}\right) \pi_{n k_{n}}=\pi_{11}+\pi_{12}+\cdots+\pi_{1 k_{1}}$

or

$$
p_{11} k_{1} \pi_{11}+p_{21} k_{2} \pi_{21}+\cdots+p_{n 1} k_{n} \pi_{n 1}=k_{1} \pi_{11}
$$

Let $k_{1} \pi_{11}=\pi_{1}, k_{2} \pi_{21}=\pi_{2}, \cdots, k_{n} \pi_{n 1}=\pi_{n}$. Note that $\pi_{i}$ is the sum of probabilities of substates in state $i$, and is therefore the probability of state $i$. Thus we get $p_{11} \pi_{1}+p_{21} \pi_{2}+\cdots+$ $p_{n 1} \pi_{n}=\pi_{1}$. Multiplying out the next $k_{2}$ rows of (1) and adding, and so forth, we can get $p_{12} \pi_{1}+p_{22} \pi_{2}+\cdots+p_{n 2} \pi_{n}=$ $\pi_{2}, \cdots, p_{1 n} \pi_{1}+p_{2 n} \pi_{2}+\cdots+p_{n n} \pi_{n}=\pi_{n}$

and the system of equations simplifies to

$$
\left[\begin{array}{ccccc}
p_{11} & p_{21} & p_{31} & \cdots & p_{n 1} \\
p_{12} & p_{22} & \cdots & & p_{n 2} \\
p_{13} & \cdots & & & \\
\vdots & & & & \vdots \\
p_{1 n} & p_{2 n} & \cdots & & p_{n n}
\end{array}\right]\left[\begin{array}{c}
\pi_{1} \\
\pi_{2} \\
\pi_{3} \\
\vdots \\
\pi_{n}
\end{array}\right]=\left[\begin{array}{c}
\pi_{1} \\
\pi_{2} \\
\pi_{3} \\
\vdots \\
\pi_{n}
\end{array}\right]
$$

where $p_{i j}$ is the sum of probabilities of any particular substate in state $i$ changing to all substates in $j$, and $\pi_{i}$ is the stationary probability of the system in state $i$.

To find $p_{i j}$, we can take a particular substate of state $i$, say $\left(i_{1} i_{2} \cdots i_{N}\right)$, and find the probability that it will change to a substate of $j$, say $\left(j_{1} j_{2} \cdots j_{N}\right)$. This probability can be found by using $p\left(i_{1} \rightarrow j_{1}, i_{2} \rightarrow j_{2}, \cdots, i_{N} \rightarrow j_{N}\right)=$ $p\left(i_{1} \rightarrow j_{1}\right) \cdot p\left(i_{2} \rightarrow j_{2} \mid i_{1} \rightarrow j_{1}\right) \cdots p\left(i_{N} \rightarrow j_{N} \mid i_{1} \rightarrow j_{1}, \cdots, i_{N-1} \rightarrow\right.$ $\left.j_{N-1}\right)$. If we write $p_{k}(i, j)=p\left(i_{k} \rightarrow j_{k} \mid\right.$ all modes $R_{m}, m<k$ already considered), then we get

$$
p_{k}(i, j)=\left\{\begin{array}{cc}
0 & j-i<-1 \\
i p(1-p)^{i-1} & j-1=-1 \\
\left\{\begin{array}{c}
P\left(\text { user } k \text { in } T_{0}\right)\left(\begin{array}{c}
r_{k}-1 \\
1
\end{array}\right) \frac{s}{N-1} \\
\left.+P\left(\text { user } k \text { not in } T_{0}\right)\left(\begin{array}{c}
r_{k} \\
1
\end{array}\right) \frac{s}{N-1}\right\}(1-p)^{i}
\end{array}\right. & j-i=0 \\
\text { or }\left\{1-i p(1-p)^{i-1}\right\} & j-i=1 \text { and } i=0 \\
\left\{\begin{array}{c}
P\left(\text { user } k \text { in } T_{0}\right)\left(\begin{array}{c}
r_{k}-1 \\
1
\end{array}\right) \frac{s}{N-1} \\
\left.+P\left(\text { user } k \text { not in } T_{0}\right)\left(\begin{array}{c}
r_{k} \\
1
\end{array}\right) \frac{s}{N-1}\right\}\left(1-(1-p)^{i}\right)
\end{array}\right. & j-i=1 \text { and } i \neq 0 \\
& \\
P\left(\text { user } k \text { in } T_{0}\right)\left(\begin{array}{c}
r_{k}-1 \\
j-i
\end{array}\right) \cdot\left(\frac{s}{N-1}\right)^{j-i} & j-i>1
\end{array}\right.
$$

where $\left(\begin{array}{l}n \\ r\end{array}\right)=n ! / r !(n-r) !$ which is the number of ways of choosing $r$ objects from $n$, and $r_{k}$ is the remaining idle users at the time $R_{k}$ is considered. Note that $p_{N}(i, j)$ involves the extra factor $(1-s)^{r_{N+1}}$ which is the probability that all unaffected stations do not transmit at all. Also $r_{k+1}=\left(r_{k}-\right.$ number of newly transmitted stations), and $p_{k}(i, j)=0$ if $r_{k+1}<0$. Then $p_{i j}=\Sigma_{\text {all substates } \epsilon j} \Pi_{k=1}^{N} p_{k}(i, j)$.

Lastly there remains only the calculation of the probability that user $k$ is in $T_{0}$. To do so, we first find all the possible configurations for a substate, and then the fraction of those configurations that has user $k$ in $T_{0}$. Since all the configurations are equally likely, the ratio of configurations of a substate with user $k$ idle to all configurations for that substate is the probability that user $k$ is idle.

Consider the substate $m=\left(m_{1} m_{2} \cdots m_{N}\right)$. If we lay out the users in the various modes in a row we get 


$$
\begin{array}{cccccc}
R_{1} & R_{2} & R_{3} & \cdots & R_{N} & T_{o} \\
X X . . X & X . . X & X . . X & & X . . X & X X . . X \\
m_{1} & m_{2} & m_{3} & \cdots & m_{N} & m_{o}
\end{array}
$$

The total number of sequences without restrictions is $N$ !. But user one cannot be in $R_{1}$, so we need to subtract $m_{1}(N-1)$ ! sequences. Similarly user 2 cannot be in $R_{2}$, etc., so we need to subtract $\left(m_{1}+m_{2}+\cdots+m_{N}\right) \cdot(N-1)$ ! sequences. Now we have oversubtracted as some of these sequences are the same, for example, those with users one and two in modes $R_{1}$ and $R_{2}$ respectively, so we need to add them back. The number of sequences with 2 particular users in their own blocked modes is $\left(m_{1} m_{2}+m_{1} m_{3}+\cdots+m_{2} m_{3}+m_{2} m_{4}+\cdots+m_{N-1} m_{N}\right) \cdot(N-2)$ !.

Now we have overadded as some of these sequences have 3 stations in their own 3 blocked modes. The number that do is $\left(m_{1} m_{2} m_{3}+m_{1} m_{3} m_{4}+\cdots+m_{2} m_{3} m_{4}+\cdots+\right.$ $\left.m_{N-2} m_{N-1} m_{N}\right) \cdot(N-3)$ !. By carrying out this line of reasoning till the end, we find that the number of possible sequences is $N !-\left(\sum m_{i}\right) \cdot(N-1) !+\left(\sum_{i \neq j} m_{i} m_{j}\right) \cdot(N-2) !-\cdots+$ $(-1)^{N} \cdot\left(m_{1} m_{2} \cdots m_{N}\right)$. To obtain the number of possible configurations, we need to weed out those sequences with the same stations in a mode. The number of sequences with $m_{1}$ stations in $R_{1}$ that are the same is $m_{1}$ ! since $m_{1}$ stations can be arranged $m_{1}$ ! ways. This is true for $m_{2}$, etc. Thus the number of possible configurations, $\beta$, is

$$
\beta=\frac{N !-\left(\sum m_{i}\right) \cdot(N-1) !+\cdots+(-1)^{N}\left(m_{1} m_{2} \cdots m_{N}\right)}{m_{1} ! m_{2} ! \cdots m_{N} !\left(N-\sum m_{i}\right) !}
$$

To find the number of configurations with the station $k$ in $T_{0}$, denoted by $\beta_{k}$, we first note that if all stations are idle, then $\beta_{k}=1$, and if all stations are blocked, $\beta_{k}=0$. If $m$ stations, $0<m<N$, are free, then we can find $\beta_{k}$ by first taking out user $k$ and putting it in $T_{0}$. By using the same line of reasoning we have used before, and keeping in mind that we are now working with only $N-1$ users since user $k$ has been put in $T_{0}$ we get

$$
\beta_{k}=\frac{(N-1) !-\left(\sum_{i \neq k} m_{i}\right) \cdot(N-2) !+\cdots+(-1)^{N} \Pi_{i \neq k} m_{i}}{m_{1} ! m_{2} ! \cdots m_{N} !\left(N-\sum_{\text {all } i} m_{i}-1\right) !}
$$

Then $P\left(\right.$ user $k$ in $\left.T_{0}\right)=\beta_{k} / \beta$.

\section{Results}

By using the system of equations (3), together with the normalizing condition $\sum_{i=1}^{n} \pi_{i}=1$, we can find the stationary probabilities of all the states $\left(\pi_{1}, \pi_{2}, \cdots, \pi_{n}\right)$. The throughput for channel $k$ of $\{i, j\}$ is given by

$$
\begin{aligned}
& S_{k}\left(\pi_{i j}\right)=P\left(k \text { in } T_{0} \mid\{i, j\}\right) {\left[\left(\begin{array}{c}
n_{0}-1 \\
1
\end{array}\right) \frac{s}{N-1}\left(1-\frac{s}{N-1}\right)^{n_{0}-2}(1-p)^{n_{k}}+\right.} \\
&\left.\left(1-\frac{s}{N-1}\right)^{n_{0}-1}\left(\begin{array}{c}
n_{k} \\
1
\end{array}\right) p(1-p)^{n_{k}-1}\right]+ \\
& P\left(k \text { not in } T_{0} \mid\{i, j\}\right)\left[\left(\begin{array}{c}
n_{0} \\
1
\end{array}\right) \frac{s}{N-1}\left(1-\frac{s}{N-1}\right)^{n_{0}-1}(1-p)^{n_{k}}+\right. \\
&\left.\left(1-\frac{s}{N-1}\right)^{n_{0}}\left(\begin{array}{c}
n_{k} \\
1
\end{array}\right) p(1-p)^{n_{k}-1}\right]
\end{aligned}
$$

The throughput for $\{i, j\}$ for the system is therefore $S\left(\pi_{i j}\right)=$ $\sum_{\text {all } k} S_{k}\left(\pi_{i j}\right) \quad$ and $\quad S\left(\pi_{i}\right)=\sum_{\text {all } j} \pi_{i j} \sum_{\text {all } k} S_{k}\left(\pi_{i j}\right)=$ $\pi_{i} \Sigma_{\text {all } k} S_{k}\left(\pi_{i j}\right)$. The total throughput for the system is thus

$$
S_{\text {total }}=\sum_{\text {all } i} S\left(\pi_{i}\right)
$$

Once the throughput is known, then by using Little's result, the total delay is easily found to be

$$
D=\frac{b}{S_{\text {total }}}
$$

where $b$ is the average number of blocked stations, $b=$ $\Sigma_{\text {all } i} \pi_{i} \Sigma_{\text {all } k}\left(n_{k}\right.$ for state $\left.i\right)$. Fig. 1 shows the throughput delay curves with $s$ varying from 0.1 to 0.9 for four different values of $p$, for a CPA LAN with 5 stations, together with some simulation points.

At $s=0.1$, throughput for all cases is about 0.5 since all the stations can transmit with ease. The delay is high for $p=0.1$ as the retransmission probability is low. The delay drops for higher $p$, but goes up again after $p=0.5$ because of frequent collisions during retransmission. This effect can be seen clearly on the $p=0.7$ curve as the delay rises most steeply. However, as $s$ increases further, average delay drops as all stations get blocked most of the time except for one, and this can then transmit successfully at every try. This effect appears as a small kink on the $p=0.5$ curve, and the much more noticeable kink on the $p=0.7$ curve. 


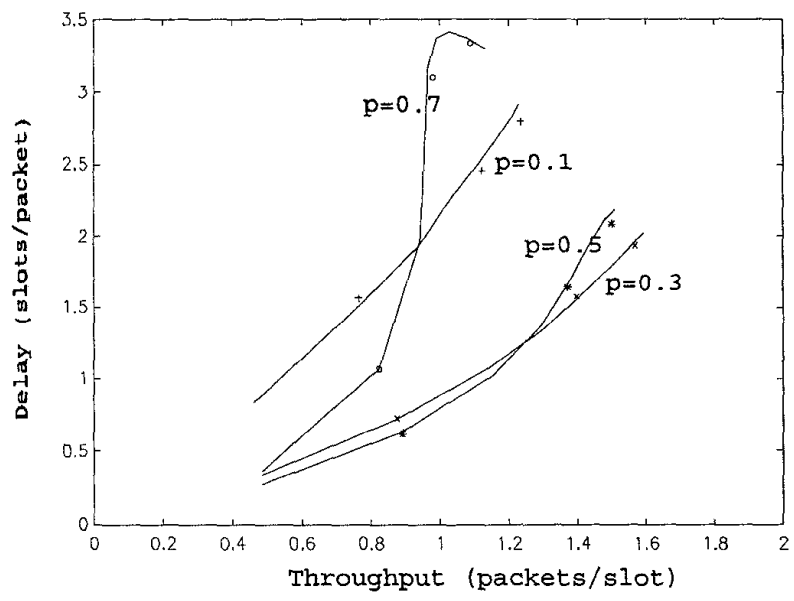

Fig. 1: Delay-Throughput characteristics for $p=0.1, p=0.3$, $p=0.5$ and $p=0.7$ together with simulation points for $s=0.2$, $s=0.5$ and $s=0.8$.

\section{CONCLUSIONS}

The low average delay experienced by the packets even when the 5-station CPA LAN is heavily loaded is deceptive, since most of the time only the packets from a single station suffer from no delay, and packets from the other stations all suffer from very large delay. However, the real strength of CPA LAN is not just the better throughput and delay characteristic when compared to slotted ALOHA. CPA LANs are very suitable for use in laboratories, hospitals, factory floors or offices where there are other machines which uses or produces narrow band radio signals. When the CPA LAN is lightly loaded, the sparsely occupied spread-spectrum channel will have a very low power/ $\mathrm{Hz}$ signal profile, and will not interfere with or be disturbed by the other machines.

The analysis given here can also be applied to spread-spectrum systems using receiver-based code protocols $([6],[7])$, where each receiver uses a different spreading code.

\section{ACKNOWLEDGMENT}

This work was supported by the HKRGC and the CRCG of The University of Hong Kong, Hong Kong.

\section{REFERENCES}

[1] W.C.Y. Lee "Overview of cellular CDMA," IEEE Trans. Vehicular Tech., Vol. VT-40, pp. 291-302, 1991.

[2] D. Makrakis, K.M.S. Sundara Murthy "Spread Slotted ALOHA Techniques for Mobile and Personal Satellite Communication Systems," IEEE J. on Select. Areas on Commun., Vol. SAC-10, pp. 9851002, 1992.

[3] K.W. Yip, T.S. Ng "Code Phase Assignment - A Technique for High Capacity Indoor Mobile DS-CDMA Communication", IEEE 44th Vehicular Tech. Conf., pp. 1586-1590, 1994.

[4] A. Alexandridis et. al, "Use of Synchronous Code Division Multiple Access Technique with Short Coding Sequences in the Development of a Local Communication Network," Int J Electronics, Vol. 68, pp. 939-956, 1990.

[5] N. Abramson "Multiple Access in Wireless Digital Networks," Proc. IEEE, Vol. 82, pp. 1360-1370, 1994.

[6] D.P. Gerakoulis, T.N. Saadawi, D.L. Schilling "A Channel Access Protocol for Embedding CSMA on Spread-Spectrum Packet Radio Networks," IEEE Int. Conf. Commun. '88, pp. 199-203, 1988.

[7] E.S. Sousa, J.A. Silvester "A Spreading Code Protocol for a Distributed Spread-Spectrum Packet Radio Network," IEEE Trans. Commun., Vol. COM36, pp. 272-281, 1988. 